\title{
Commentary
}

See article on page 522

\section{Ulcerative colitis: sclerosing cholangitis today, cancer tomorrow?}

The relation between inflammatory bowel disease (IBD), mainly ulcerative colitis, and primary sclerosing cholangitis (PSC) is particularly close, as approximately two thirds of patients with PSC have, or will develop, ulcerative colitis, and PSC occurs in approximately $3-7 \%$ of patients with ulcerative colitis. In a large Swedish epidemiological study, approximately $5.5 \%$ of patients with extensive colitis had PSC. ${ }^{1}$ Despite the close relation the aetiology of both diseases is unknown.

It is well established that patients with ulcerative colitis have an increased risk of developing colorectal carcinoma. The two major risk factors that have been identified in the development of neoplasia are duration of disease and the extent of colitis. Additional risk factors have been described such as the presence of colonic epithelial cell dysplasia and early onset of colitis. It has been suggested that pharmacological treatment for ulcerative colitis may reduce the risk of colorectal cancer. ${ }^{2}$

Recently, the presence of PSC has been suggested to increase the risk of dysplasia and colorectal cancer in patients with ulcerative colitis. ${ }^{3}$ In the initial reports from Sweden the absolute cumulative risk of developing colorectal dysplasia/carcinoma in the PSC/ulcerative colitis group was $9 \%, 31 \%$ and $50 \%$, respectively after 10,20 and 25 years of disease duration. In the group with ulcerative colitis only, the corresponding risk was $2 \%, 5 \%$ and $10 \%$, respectively. ${ }^{3}$ Moreover, 10 of the patients with PSC developed cholangiocarcinoma, all but one having ulcerative colitis, whereas in the control group of patients with ulcerative colitis, no cholangiocarcinoma occurred. This suggests that patients with PSC have an increased neoplastic potential to develop cholangiocarcinoma and colon cancer. These findings have been confirmed by a number of other studies from North America. ${ }^{45}$ However, conflicting data were provided by the Mayo Clinic. ${ }^{6}$ Loftus et al pointed out that previous studies have been limited by small sample size and the possibility of referral bias. They identified 178 patients with PSC who were stratified by geographical area of residence and were followed up to determine survival and development of colorectal cancer. ${ }^{6}$ As expected, the relative risk of colorectal cancer was increased 10-fold in those patients who had PSC and ulcerative colitis, but there was no increased risk in patients who had PSC alone. ${ }^{6}$ The number of cancers observed was similar to those expected in patients with ulcerative colitis.

The problems with assessing the risk of PSC in ulcerative colitis is that the clinical course of the colitis differs from other patients with extensive ulcerative colitis alone. ${ }^{7}$ In PSC, although total in extent, the clinical course of the colitis is often quiescent and, indeed, may only be identified by colonoscopic biopsy in patients who are, and may remain, asymptomatic. The onset of the colitis is therefore very hard to determine and duration of the disease may be underestimated, which may be an important factor when comparing with control patients with ulcerative colitis alone. This factor is emphasised by the finding of a subclinical time span of IBD in patients with PSC of as long as seven years. ${ }^{8}$

In this issue (see page 522) Kornfeld et al again tackled the question of whether there is an increased risk of colorectal cancer in patients with ulcerative colitis and PSC. This large study has the benefit of being based on a population in which all patients with a cholangiographic picture of PSC were included. However, a control group was not studied and comparisons are made with previous studies in which the risk of colorectal cancer in ulcerative colitis has been assessed. In none of these studies, however, has a number of patients with PSC been presented and it is possible, indeed likely, that some patients in these groups did have co-existing PSC. Kornfeld et al's study has the advantage of being large, including a background study population of almost one million inhabitants, from which 125 patients with PSC were identified. The number of patients with PSC and IBD is only $68 \%$, which is a lower figure compared with other Scandinavian studies, and is unclear from the study whether patients without IBD underwent colonoscopy. The diagnosis of colorectal cancer was based on data obtained from the Swedish National Cancer Registry which has the advantage of including the total number of colorectal cancers in Sweden. The use of colorectal cancer and not colonic dysplasia as the outcome measure is an advantage of the study because of the reproducibility of the diagnosis of colonic carcinoma; in addition, registrations of cancer are carefully documented in Sweden. However, as it is well recognised that epithelial low and high grade dysplasia are premalignant lesions, and that surveillance programmes for ulcerative colitis are widespread in Sweden, several patients with PSC and ulcerative colitis may have undergone colectomy before the development of cancer. The number of patients developing colorectal cancer in extensive ulcerative colitis is dependent, therefore, on the number of patients included in colonoscopic surveillance programmes. Furthermore, the number of patients developing colorectal cancer depends on the overall colectomy rate. It is possible that the quiescent cause of colitis in PSC may lead to a lower frequency of colectomies due to activity related factors in patients with ulcerative colitis alone. Another possibility suggested by the authors is that the increased number of colorectal cancers in PSC could be due to a lower frequency of prophylactic treatment with sulphasalazine among patients with PSC as they generally have a mild colitis, but this issue remains speculative as it was not documented in the results. It has been suggested that the increased use of immunosuppressive treatment in patients with PSC who receive a liver transplant explains the increased risk of colorectal cancer seen in patients with PSC and ulcerative colitis after liver transplantation. ${ }^{9}$ Such confounding variable factors are not considered by Kornfeld et al.

These results provide further evidence that there is an increased risk for colorectal cancer in patients with ulcerative colitis and PSC. It is unclear whether these patients 
should be offered colonoscopic surveillance at shorter time intervals than patients with ulcerative colitis alone. The challenge for the future is to clarify why the former seem to have an increased risk of developing both colorectal carcinoma and cholangiocarcinoma, in order to pinpoint factors which may identify patients who are at particular risk and in whom prophylactic surveillance and treatment might be indicated.

Department of Gastroenterology/Hepatology,

ULRIKA BROOMÉ

Huddinge University, Sweden

R W CHAPMAN

Department of Gastroenterology,

fohn Radcliffe Hospital,

Oxford, UK

1 Olsson R, Danielsson Å, Järnerot G, Lindström E, Lööf L, Rolny P, et al. Prevalence of primary sclerosing cholangitis in patients with ulcerative colitis. Gastroenterology 1991; 100: 1319-23.
2 Pinczowski D, Ekbom A, Baron J, Yuen J, Adami H-O. Risk factors for colorectal cancer in patients with ulcerative colitis: a case control study. Gastroenterology 1994; 107: 117-20.

3 Broomé U, Löfberg R, Veress B, Eriksson LS. Primary sclerosing cholangitis and ulcerative colitis: evidence for increased neoplastic potential. Hepatology 1995; 22: 1404-8.

4 Brentnall TA, Haggitt RC, Rabinovitch PS, Kimmey MB, Bronner MP, Levine DS, et al. Risk, and natural history of colonic neoplasia in patients with primary sclerosing cholangitis and ulcerative colitis. Gastroenterology with primary scler

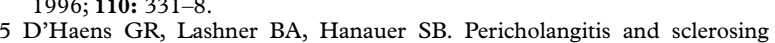
cholangitis are risk factors for dysplasia and cancer in ulcerative colitis. Am f Gastroenterol 1993; 88: 1174-8.

6 Loftus EV, Sandborn WJ, Tremaine J. Mahoney DW, Zinsmeister AR, Offord KP, et al. Risk of colorectal neoplasia in patients with primary sclerosing cholangitis. Gastroenterology 1996; 110: 432-40.

7 Lundqvist K, Broomé U. Differences in colonic disease activity in patients with ulcerative colitis with and without primary sclerosing cholangitis. Dis Colon Rectum 1997; 40: 1-6.

8 Broomé U, Löfberg R, Lundqvist K, Veress B. Subclinical time span of inflammatory bowel disease in patients with primary sclerosing cholangitis. Dis Colon Rectum 1995; 38: 1301-5.

9 Higashi H, Yanaga K, Marsh JW, Tzakis A, Kakizoe S, Starzl TE. Development of colon cancer after liver transplantation for primary sclerosing cholangitis associated with ulcerative colitis. Hepatology 1990; 11: 477-80. 